\title{
EFFECT OF THE OSCILLATORY SHAPE OF THE EARTH STATION ANTENNA RADIATION PATTERN IN THE AGGREGATE INTERFERENCE GENERATED BY NON-GSO SATELLITE SYSTEMS
}

\author{
José Mauro P. Fortes and Raimundo Sampaio-Neto
}

\begin{abstract}
This paper focuses on interference aspects related to non-GSO satellite systems and presents results reflecting the effect of the oscillatory shape of the earth station antenna sidelobe gain pattern in the statistical behaviour of the aggregate interference produced by an interfering non-GSO system into the satellites of an interfered-with system. A Bessel function type of radiation pattern was adopted as a more realistic model for the antenna sidelobe gain since it reflects the oscillatory behaviour encountered in measured radiation patterns. This sidelobe gain oscillatory behaviour plays an important role when addressing interference calculations involving non-GSO systems, since due to the non-GSO system satellites dynamics not all entries in the aggregate interference are associated with the maximum sidelobe earth station antenna gain. Results indicate that, although often used in GSO satellite systems, this type of worst case interference calculation tends to be overly pessimistic if applied to the non-GSO satellite environment.
\end{abstract}

Keywords: Non-geoestacionary systems, interference, antenna, sidelobe emissions.

Resumo - Este trabalho aborda aspectos de interferência em sistemas de comunicações que utilizam satélites nãogeoestacionários (non-GSO). Em particular, considera-se o efeito que a característica oscilatória do diagrama de radiação dos lobos laterais das antenas das estações terrenas têm no comportamento estatístico da interferência agregada produzida pelas estações de um sistema non-GSO interferente nos satélites de um outro sistema non-GSO. Um diagrama de radiação modelado com funções de Bessel foi adotado aqui para o ganho dos lobos laterais das antenas, já que ele reflete de forma adequada as variações deste ganho presentes em diagramas de radiação obtidos experimentalmente. A variação acentuada do ganho dos lobos laterais tem um impacto significativo nos cálculos de interferência envolvendo sistemas non-GSO, já que, devido às dinâmicas dos satélites destes sistemas, nem todas as componentes da interferência agregada estão associadas permanentemente ao maior valor de ganho dos lobos laterais das antenas da estação interferente. Os resultados obtidos indicam que, apesar de freqüentemente utilizado em situações envolvendo satélites geoestacionários, este tipo de cálculo de interferência de pior caso tende a ser

Authors are with Pontifícia Universidade Católica do Rio de Janeiro, Rio de Janeiro, RJ, Brazil. E-mails: \{jmfortes, raimundo\}@cetuc.puc-rio.br. Review coordinated by Denise Consonni (Area Editor). Manuscript received May/29/2002, reviewed Aug/01/2003, accepted Aug/19/2003. demasiadamente pessimista se aplicado ao caso de sistemas non-GSO . Este fato indica que as antenas das estações terrenas que operam com satélites non-GSO podem ter restrições de projeto substancialmente menos exigentes do que as que operam com satélites geoestacionários.

Palavras-chave: Sistemas non-GSO, Interferências, antenas, emissão pelos lóbulos laterais.

\section{INTRODUCTION}

Interference calculations involving GSO satellite systems are usually based on the assumption that the earth station sidelobe antenna gain is equal to an envelope of the form $x-25 \log \theta$. However, the oscillatory behaviour encountered in measured radiation patterns plays an important role when addressing interference calculations involving non-GSO systems, since due to the non-GSO system satellites dynamics not all entries in the aggregate interference are associated with the maximum sidelobe earth station antenna gain represented by its envelope. This paper considers two non-GSO satellite systems, here referred to as LEO 1 and LEO 2, and addresses the more critical case of up-link interference, in which the number of earth stations and their geographical distribution play an important role. The effect of the oscillatory shape of the earth station antenna sidelobe gain in the cumulative distribution functions of the carrier to aggregate interference ratio is evaluated considering the up-link interference from LEO 1 earth stations into a LEO 2 satellite. To obtain accurate interference statistics, full constellation of the interfering system is considered. Furthermore, multiple earth station locations (up to 120 earth stations for LEO 1), with multiple earth station antenna beams (up to 4) are assumed. The results were obtained through the analytical/numerical method described in [1,2]. As pointed out in [2], if compared to computer simulation the results generated by this analytical method correspond to an infinite number of simulated days, and therefore the method does not suffer from the need for long running times as may be required in computer simulation methods to assure statistically significant results.

The methodology and the interference model used in the interference computations are described in Section 2, where a Bessel function type of radiation pattern is adopted as a more realistic model for the antenna sidelobe gain since it reflects the oscillatory behaviour encountered in measured radiation patterns. This section also contains a brief description of the algorithm developed to determine the worst location for 
an interfered-with satellite in the victim non-GSO system. Numerical results are presented in Section 3. These results illustrate the effects of the earth station antenna sidelobe gains and of the number of interfering earth stations in the up-link $C / I$ cumulative distribution function. This type of result is important for the establishment of design objectives for the antennae of earth stations operating with non-GSO satellites. Finally, main conclusions are highlighted in Section 4.

\section{METHODOLOGY}

As mentioned before the computation of the probability distribution of the carrier to aggregate interference ratio was based on the analytical/numerical method described in $[1,2]$. The situation involving up-link interference from LEO 1 earth stations into LEO 2 satellites is illustrated in Figure 1. In this figure, each earth station is assumed to have four antennae (beams), pointed to the LEO 1 satellites corresponding to the four highest elevation angles that satisfy the minimum elevation angle constraint. Considering that all feeder link earth stations transmit the same power, the aggregate up-link interference power reaching a LEO 2 satellite (say, satellite $i$ ), located at a given point, is proportional to the quantity

$$
z_{i}=\sum_{j=0}^{N_{+}-1} \sum_{k=0}^{N_{a}-1} \frac{G_{s . i}\left(\alpha_{i j}\right) G_{e . j}\left(\beta_{i j k}\right)}{d_{i j}^{2}}
$$

where $G_{s . i}\left(\alpha_{i j}\right)$ is the receiving antenna gain of satellite $i$ in a direction $\alpha_{i j}$ degrees off the main beam axis, $G_{e, j}\left(\beta_{i j k}\right)$ is the earth station transmitting antenna gain in a direction $\beta_{i j k}$ degrees off the main beam axis and $d_{i j}$ is the range between satellite $i$ and the earth station $j$. Note that the random variable $\hat{s}_{i}$ is a function of the given location of the considered LEO 2 interfered-with satellite and the random location of the LEO 1 reference satellite (see $[1,2]$ ). The integers $N_{e}$ and $N_{a}$ represent, respectively, the number of earth stations and the number of antennae (per earth station) tracking a LEO 1 satellite with an elevation angle higher than the prescribed minimum value.

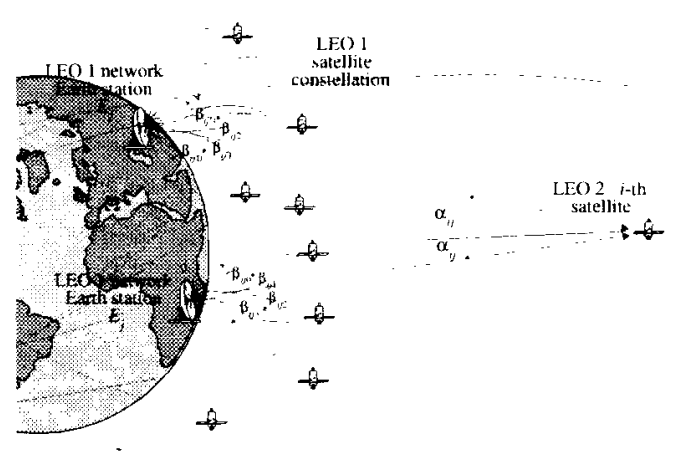

Figure 1. Up-link interference geometry.

The following assumptions were made in the interference computations:

\subsection{SYSTEMS PARAMETERS}

The LEO 1 and LEO 2 system characteristics used here were assumed to be respectively equal to those for LEO D and LEO F in Recommendation ITU-R S.1328-2 (year 2000 version) [3], except for the following parameters:

- the minimum operating elevation angle for the LEO 1 earth stations was assumed to be 5 degrees (instead of the 10 degrees in Recommendation ITU-R S.1328-2);

- the LEO 2 satellite antenna radiation pattern was assumed to be the one in Annex 1 to Appendix 30B of the Radio Regulations [4], with maximum gain set to 13 $\mathrm{dB}$ for transmission and $12 \mathrm{~dB}$ for reception. In both cases the half-power beamwidth was 26 degrees;

- the LEO 1 transmitting and receiving satellite antenna radiation patterns were both given by the curve in Figure 2;

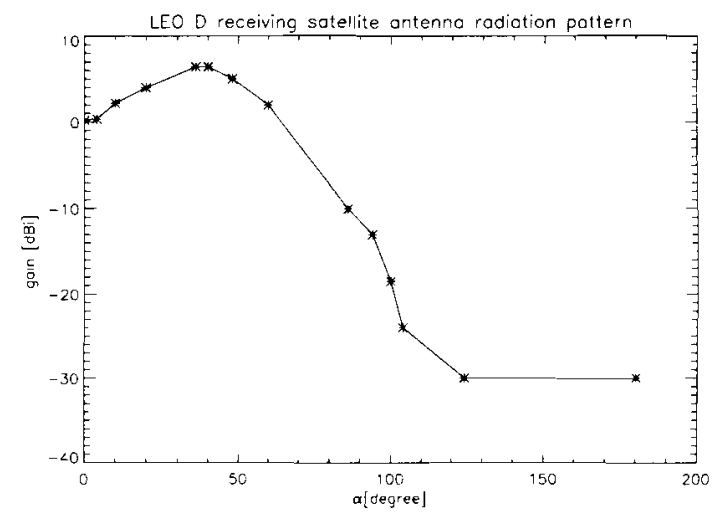

Figure 2. LEO 1 satellite antenna radiation pattern (transmitting and receiving)

Concerning the earth station switching strategies, it was assumed that each LEO 1 gateway contains four ES antennae that track LEO 1 satellites with elevation angles higher than the prescribed minimum value.

\subsection{INTERFERED-WITH SATELLITE LOCA- TION (WORST CASE)}

For the purpose of interference calculations, the location of the interfered-with satellite (LEO 2) was determined based on a worst case criterion. An algorithm was developed to determine the distribution, over the whole spherical surface containing the satellites of the interfered-with system (system "shell"), of the "in-line" interference levels produced by all earth stations tracking visible satellites in the interfering system, averaged with respect to the random location of the interfering system reference satellite $[1,2]$. Next, the interfered-with system "shell" is partitioned into square regions ( 1 by 1 degree) centered at $\left\{c_{i j}=\left(\phi_{i} \theta_{j}\right)^{T} ; i=\right.$ $\left.-180, \ldots, 180, j=-\theta_{\max }, \ldots, \theta_{\max }\right\}$, and the average "inline" interference level distribution is then used to determine the total "in-line" interference $I_{I L}\left(c_{i j}\right)$ level contained inside each square region. To take into account the uncertainty in the 
location of the interfered-with satellite, the worst location is defined as the point $c_{i j}^{*}$ corresponding to the maximum value of $I_{W}\left(c_{i j}^{*}\right)=I_{I L}\left(c_{i j}^{*}\right) p\left(c_{i j}^{*}\right)$, where $p\left(c_{i j}^{*}\right)$ is the probability of finding a satellite of the interfered-with system inside the square region centered at $c_{i j}^{*}$. This probability was obtained from the satellite location probability density function in $[1,2]$.

Figure 3 present the results obtained with this procedure in a particular situation where 120 LEO I earth stations produce up-link interference into LEO 2 satellites and illustrate the location of the 120 LEO 1 earth stations. Figure 3 also illustrates the contour curves of the function. The black cross indicates the worst location for the LEO 2 satellite. Figure 4 illustrates an earth view from a LEO 2 satellite placed at this worst location .

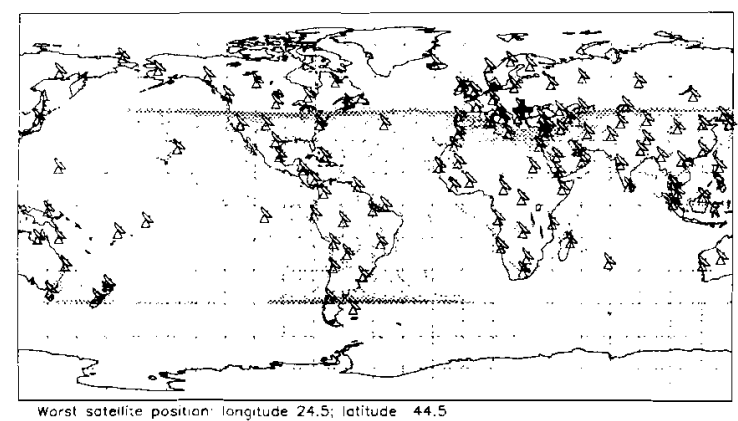

Figure 3. Contour curves of the function $I_{W}$ and worst location for the LEO 2 satellite (black cross).

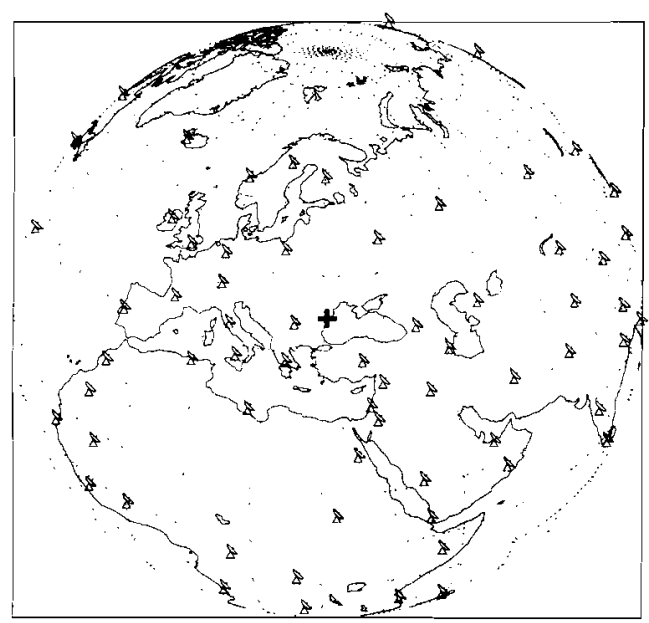

Figure 4. Earth station view from a LEO 2 satellite placed at the worst case location $c_{i j}^{*}$. Note that 65 of the 120 LEO 1 earth stations can be seen from this satellite location.

\subsection{CARRIER-TO-INTERFERENCE RATIO $(C / I)$ EXPRESSIONS}

The $C / I$ cumulative probability distribution (CDF) corresponding to a interfered-with satellite at the worst location was obtained by first determining, using the analytical method in $[1,2]$, the CDF of the random variable $z_{d B}=$ $10 \log z$, with $z$ in the form of (1). The CDF of the carrier to interference ratio $C / I$ (in $\mathrm{dB}$ ) was then obtained using the following relations:

- Interference into a LEO 2 satellite $(C / I$ within the receiver bandwidth):

$$
\left(\frac{C}{I}\right)_{[d B]}=h_{1}-z_{d B} .
$$

with

$$
\begin{aligned}
k_{1}= & (\text { e.i.r.p. })_{E S-L E O 2}+10 \log \frac{G_{s 2}(a) G_{e 1}(0)}{d^{2}} \\
& -(\text { e.i.r.p. })_{E S-L E O 1}-10 \log \frac{B_{L E O 2}}{B_{L E O 1}}, \text { (3) }
\end{aligned}
$$

where $\alpha$ is the LEO 2 satellite antenna main beam off-set angle in the direction of the LEO 2 earth station transmitting the desired carrier, $d$ the is distance between this earth station and the LEO 2 satellite, $B_{L E O} 1$ and $B_{L E O} 2$ denote, respectively, the LEO 1 and LEO 2 receiver noise bandwidth and the equivalent isotropically radiated power (e.i.r.p.) values refer to e.i.r.p. per carrier. Note that the ratio $\frac{B_{L E W 2}}{B_{L L C}}<1$ corresponds to an interference reduction factor due to the difference, in bandwidth, of the interfering and interfered-with carriers.

\subsection{EARTH STATION ANTENNA SIDELOBE RADIATION PATTERNS}

Two alternatives were considered for the antenna radiation patterns used in interference calculations. The first alternative refers to earth station antenna radiation patterns with the usual sidelobe gain of the form $x-25 \log \theta$, and is generally described by

$$
G_{e}(\theta)=\left\{\begin{array}{lc}
G_{\max }-2.5 \times 10^{-3}\left(\frac{D}{\lambda} \theta\right)^{2}, & 0 \leq \theta<\theta_{m} \\
G_{1}, & \theta_{m} \leq \theta<\theta_{r} \\
G_{1}-\frac{G_{1}-x}{1-\theta_{r}}\left(\theta-\theta_{r}\right), & \theta_{r} \leq \theta<1^{\circ} \\
x-25 \log \theta, & 1^{\circ} \leq \theta<\theta_{s} \\
-3.5, & \theta_{s} \leq \theta<\theta_{c} \\
32-25 \log \theta, & \theta_{c} \leq \theta<48^{\circ} \\
-10, & 48^{\circ} \leq \theta \leq 180^{\circ}
\end{array}\right.
$$

with

$$
G_{1}=2+15 \log \frac{D}{\lambda}
$$

and

$$
\begin{aligned}
\theta_{m} & =\frac{20 \lambda}{D} \sqrt{G_{\max }-2-15 \log \frac{D}{\lambda}} . \\
\theta_{r} & =15.85\left(\frac{D}{\lambda}\right)^{-0.6}, \\
\theta_{s} & =10^{\frac{r+3.5}{25}}, \\
\theta_{c} & =10^{\frac{32+3.5}{25}} .
\end{aligned}
$$

where $D$ represents the earth station antenna diameter and $\lambda$ the wavelength corresponding to the carrier frequency, both in the same unit. Figure 5 illustrates earth station radiation patterns with usual $[5,6,7]$ sidelobe gains of the form 
$x-25 \log \theta$ different values of $x$. The second alternative corresponds to a Besse] function radiation pattern having a sidelobe gain that shows an oscillatory behaviour similar to that encountered in measured radiation patterns. By using this radiation pattern it is possible to get closer to the actual situation in which not all entries in the aggregate interference are associated with the maximum sidelobe earth station antenna gain. The Bessel function type of antenna radiation pattern used here is a version of that presented in [8], modified to better fit an envelope $32-25 \log \theta$ which could be used as a design objective for the radiation patterns of earth stations operating with non-GSO satellites. This antenna radiation pattern is given by

$G_{e}(\theta)= \begin{cases}G_{\max }+20 \log \left(c(\theta) \frac{\left|J_{1}(u(\theta))\right|}{u(\theta)}\right), & 0 \leq \theta<48^{\circ} \\ -10, & 48^{\circ} \leq \theta \leq 180^{\circ}\end{cases}$

with

$$
u(\theta)=\frac{\pi D}{\lambda} \sin (\theta)
$$

and

$$
c(\theta)=h(\theta) f(\theta)
$$

where

$h(\theta)= \begin{cases}1, & 0 \leq \theta<\theta_{1} \\ 1+a \frac{\left(\theta-\theta_{1}\right)^{0.3}}{\sigma^{2}} \exp \left[-\frac{\left(\theta-\theta_{1}\right)^{2}}{2 \sigma^{2}}\right], & \theta_{1} \leq \theta \leq 48^{\circ}\end{cases}$

and

$$
f(\theta)=\left\{\begin{array}{lr}
2, & 0 \leq \theta<\theta_{2} \\
\frac{\mathrm{X}_{48}-2}{48-\theta_{2}}\left(\theta-\theta_{2}\right)+2, & \theta_{2} \leq \theta \leq 48^{\circ}
\end{array}\right.
$$

with

$$
X_{48}=1468.85 \times 10^{-G_{m a x} / 20}
$$

The values of the parameters $a$ and $\sigma^{2}$ are adjusted to fit the $32-25 \log \theta$ envelope and depend on the value of $G_{\max }$. This Bessel function radiation pattern is illustrated in Figure 6 for a 6 meter antenna operating in a $5.175 \mathrm{GHz}$ with $G_{\max }=$ 47.5. In this particular case $a=0.5$ and $\sigma=16$. This figure also shows the $32-25 \log \theta$ sidelobe gain radiation pattern.

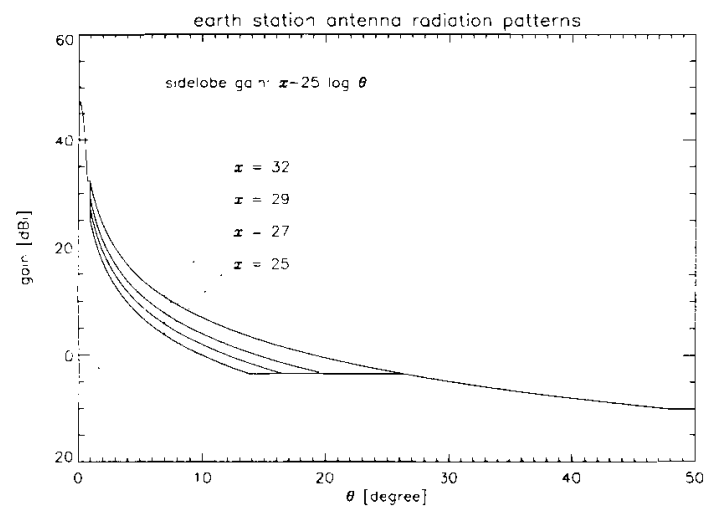

Figure 5. Earth station antenna sidelobe patterns.

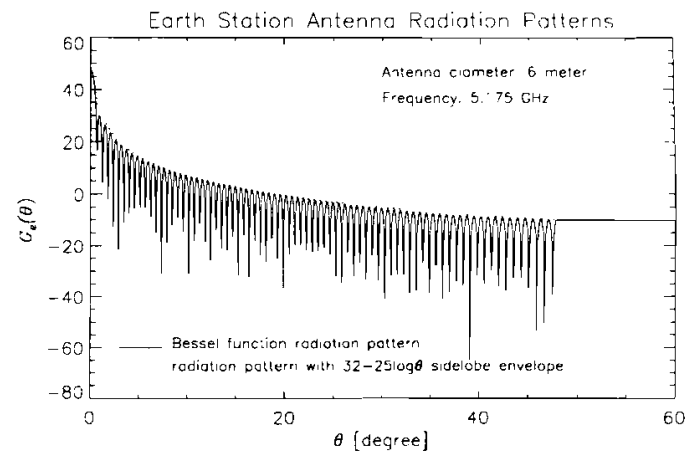

Figure 6. Bessel function antenna radiation pattern

\section{NUMERICAL RESULTS}

Interference criteria for frequency sharing are usually expressed in the form

$$
P\left(\frac{C}{I} \leq X_{i}\right) \leq p_{i} \quad ; \quad i=1, \ldots, K
$$

meaning that the $C / I$ cumulative probability distribution function (CDF) at some specific points $X_{l}$ must fall below prescribed probability values $p_{i}$. For the evaluation of the effects of the earth station antenna sidelobe gain in the statistical behaviour of the aggregate $C / I$ ratio, a total of up to 120 earth stations was considered for the up-link interference of LEO 1 system into a LEO 2 satellite. The cumulative distribution function (CDF) of the aggregate $C / I$ ratio was determined for different earth station antenna sidelobe patterns: the Bessel function pattern satisfying the $32-25 \log \theta$ envelope (with $a=0.5$ and $\sigma=16$ ) and a pattern with sidelobe gain of the form $x-25 \log \theta$, for which the value of $x$ was varied down from 32 until the resulting $C / I$ cumulative distribution function curve touched (or nearly touched) that of the Bessel function pattern.

As described in Section 2.3, the $C / I$ cumulative probability distribution (CDF) corresponding to a interfered-with satellite at a given location was obtained by first determining, using the analytical method in $[1,2]$, the CDF of the random variable $z_{d B}=10 \log z$, with $z$ in the form of (1). The CDF of the carrier to interference ratio $C / I$ (in $\mathrm{dB}$ ) was then obtained considering the relations in (2) and (3). It was assumed that the interfered-with satellite was at the worst location (see Section 2.2). Furthermore, the earth station transmitting the desired carrier was assumed to be located in such way that its transmitting antenna operates with the minimum permissible elevation angle (smallest desired carrier power $C$ ).

Performance results were obtained for a total of $n=30$, $n=60, n=90$ and $n=120$ LEO 1 earth stations. Starting with a given set of 120 locations smaller sets were obtained by eliminating station locations in such way that the relative geographical distribution was maintained. The resulting sets of earth station locations corresponding to $n=60$ and $n=$ 120 are respectively illustrated in figures 7 and 8 together with the corresponding worst location for the interfered-with LEO 2 satellite. The CDFs of the aggregate $C / I$ ratio associated with the sets of locations in figures 7 and 8 are re- 
spectively displayed, in both linear and logarithm scales, in figures 9 to 12 .

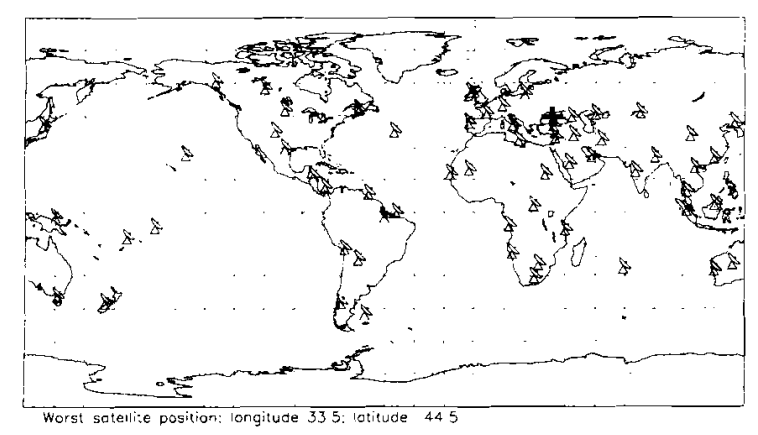

Figure 7. LEO 1 Earth station distribution and worst location for the LEO 2 satellite $(n=60)$.

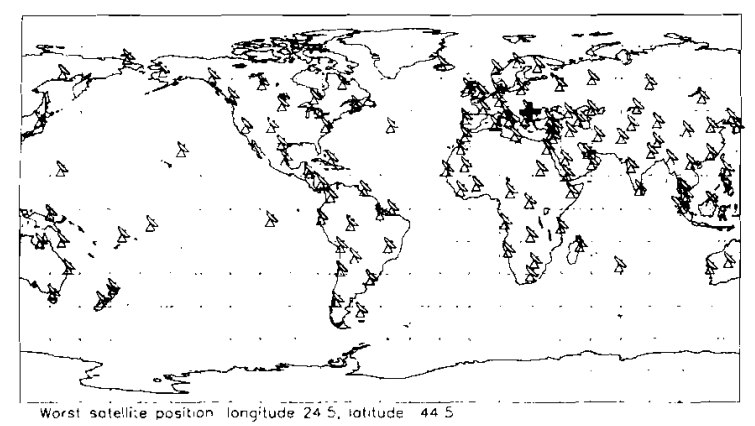

Figure 8. LEO 1 Earth station distribution and worst location for the LEO 2 satellite $(n=120$ ).

The following observations can be made based on the results in figures 9 to 12 :

- A comparison of the curves associated to the $x-25 \log \theta$ type of sidelobe gain with those corresponding to the Bessel function radiation pattern with an envelope of the form $32-25 \log \theta$ shows that the worst-case type of interference calculation that considers that all entries in the aggregate interference are associated with the maximum sidelobe antenna gain leads to results that are pessimistic even for values of $x$ as low as 25 .

- If the earth station antennae satisfy the current design objective of $29-25 \log \theta$ [6] and the usual worst-case type of interference calculation indicates that the interference criteria are met, then these same interference criteria are also met by earth station antennae that have a less stringent design objective (as for example the $32-25 \log \theta$ objective) when the oscillatory shape of the radiation pattern is taken into account. This indicates that antennae of earth stations operating with non-GSO satellites could have a design objective substantially less stringent than that of earth stations operating with GSO satellites.

The impact of increasing number of LEO 1 earth stations in a LEO 2 satellite aggregate interference is illustrated by figures 13 to 16 respectively for the Bessel function type of earth station antenna radiation pattern and the more pessimistic $32-25 \log \theta$ type of antenna sidelobe gain.
The results in figures 13 to 16 indicate that an increase in the number of interfering LEO 1 earth stations impacts more the lower aggregate interference levels since, in general, higher levels of interference correspond to "in-line" interference which are mainly dominated by interference from a few earth stations (one in most cases).

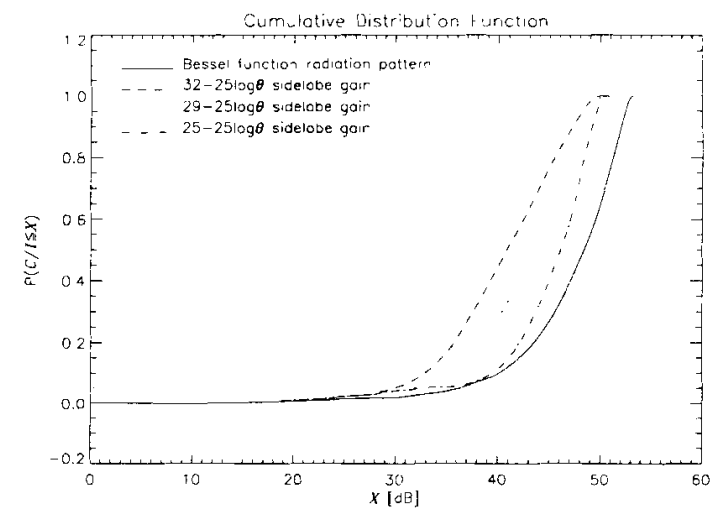

Figure 9. Aggregate $C / I$ ratio CDF corresponding to uplink interference into a LEO 2 satellite at the worst location (60 LEO 1 earth stations worldwide).

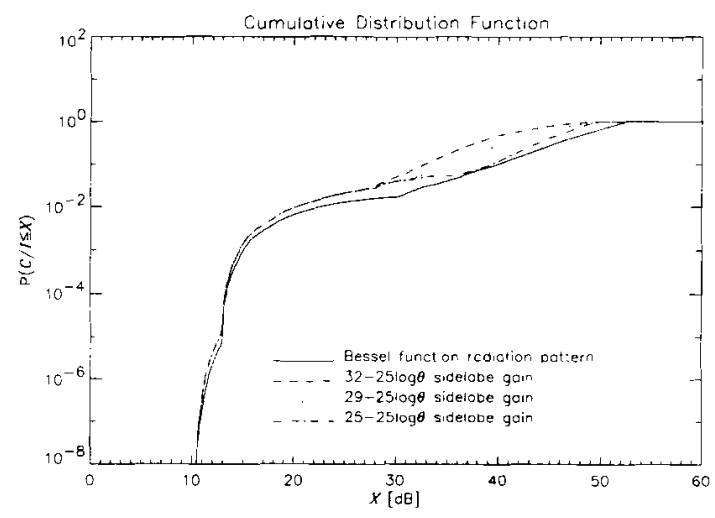

Figure 10. Expanded view of the aggregate $C / I$ ratio $\mathrm{CDF}$ corresponding to up-link interference into a LEO 2 satellite at the worst location (60 LEO 1 earth stations worldwide).

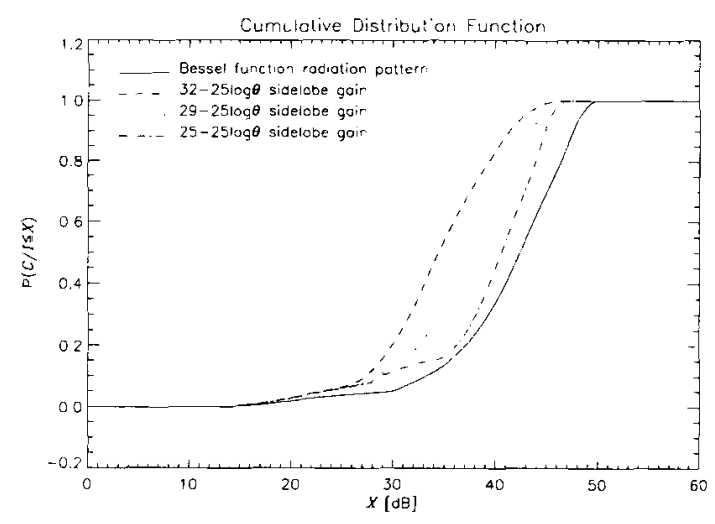

Figure 11. Aggregate $C / I$ ratio CDF corresponding to uplink interference into a LEO 2 satellite at the worst location (120 LEO 1 earth stations worldwide). 


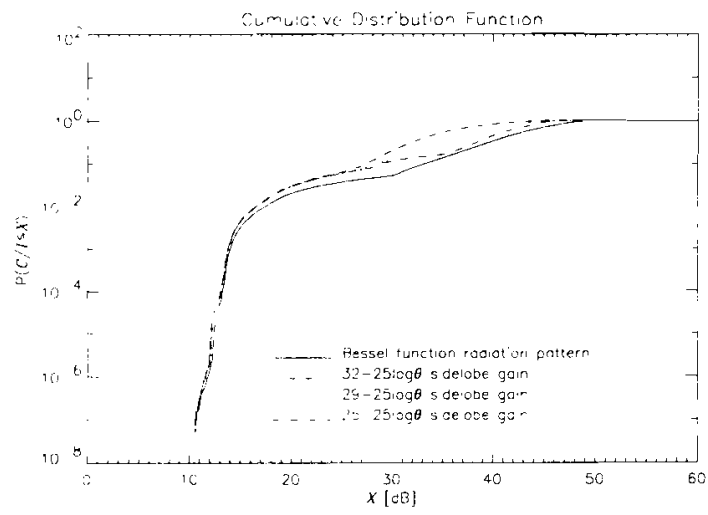

Figure 12. Expanded view of the aggregate $C / I$ ratio $C D F$ corresponding to up-link interference into a LEO 2 satellite at the worst location (120 LEO 1 earth stations worldwide).

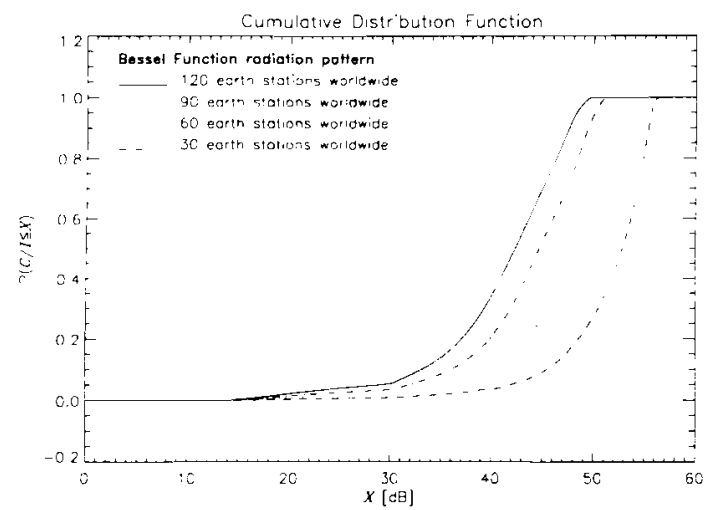

Figure 13. Aggregate $C / I$ ratio CDF corresponding to uplink interference into a LEO 2 satellite at the worst location for different number of LEO 1 earth stations(Bessel function radiation pattern).

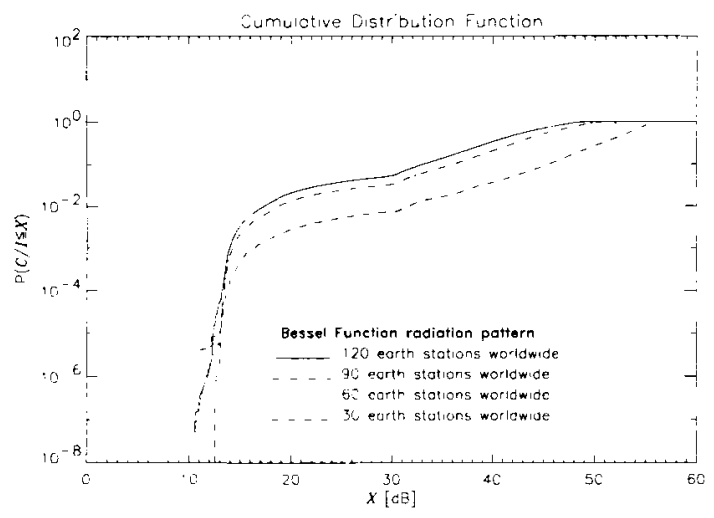

Figure 14. Expanded view of the aggregate $C / I$ ratio $\mathrm{CDF}$ corresponding to up-link interference into a LEO 2 satellite at the worst location for different number of LEO 1 earth stations(Bessel function radiation pattern).

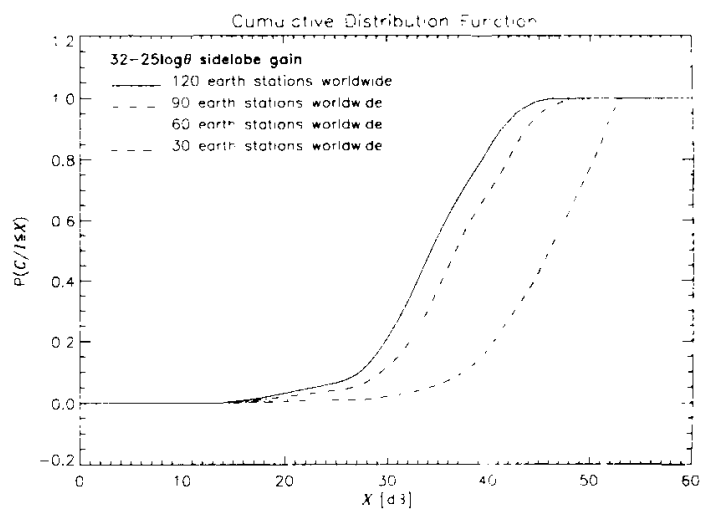

Figure 15. Aggregate $C / I$ ratio $C D F$ corresponding to uplink interference into a LEO 2 satellite at the worst location for different number of LEO 1 earth stations $(32-25 \log \theta$ antenna sidelobe gain).

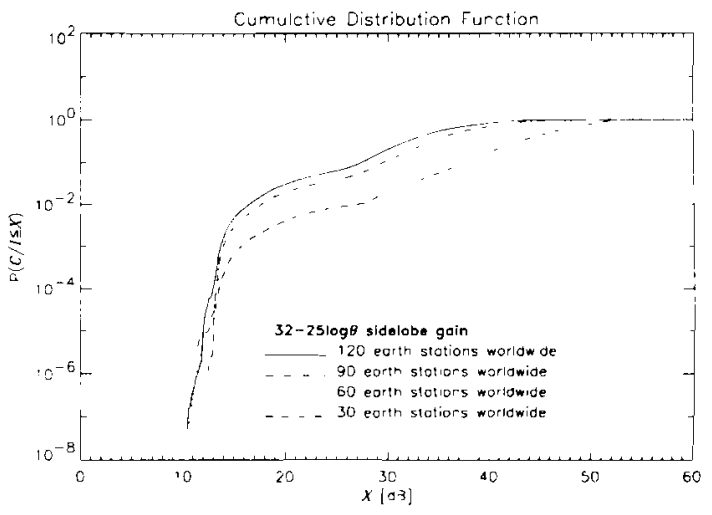

Figure 16. Expanded view of the aggregate $C / I$ ratio $C D F$ corresponding to up-link interference into a LEO 2 satellite at the worst location for different number of LEO 1 earth stations ( $32-25 \log \theta$ antenna sidelohe gain)).

\section{CONCLUSION}

The effect of the oscillatory behaviour of the earth station antenna sidelobe gain in the cumulative distribution functions for the carrier to aggregate interference ratio was evaluated for a situation involving up-link interference from LEO 1 earth stations into a LEO 2 satellite. The $C / I$ CDFs were obtained through the analytical method described in [1, 2]. A Bessel function type of radiation pattern was adopted as a more realistic model for the antenna sidelobe gain since it reflects the oscillatory behaviour encountered in measured radiation patterns.

Using the Bessel function pattern as a reference, and considering the interference situation examined, results have shown that the worst-case type of interference calculation that considers that all entries in the aggregate interference are associated with the maximum sidelobe antenna gain leads to results that are overly pessimistic when applied to the nonGSO satellite environment. Results have shown that if the earth station antennae satisfy the current design objective of 
$29-25 \log \theta[6]$ and the usual worst-case type of interference calculation indicates that the interference criteria are met, then these same interference criteria are also met by earth station antennae that have a less stringent design objective (as for example the $32-25 \log \theta$ objective) when the oscillatory shape of the radiation pattern is taken into account. This indicates that antennae of earth stations operating with non-GSO satellites could have a design objective substantially less stringent than that of earth stations operating with GSO satellites.

\section{ACKNOWLEDGEMENTS}

This work was supported by ICO Global Communications under Contract No. IGCH(BV)/97-1010

\section{REFERENCES}

[1] J. M. P. Fortes and R. Sampaio-Neto, "An Analytical Method for Assessing Interference in an Environment Involving NGSO Satellite Networks," in Proceedings of IEEE/SBT International Telecommunications Symposium, São Paulo, Brazil, Aug. 1998, vol. 1, pp. 5-9.

[2] J. M. P. Fortes and R. Sampaio-Neto, "An Analytical Method for Assessing Interference in Interference Environments Involving NGSO Satellite Networks," International Journal of Satellite Communications, vol. 17 No. 6, NovemberDecember 1999.

[3] "Satellite System Characteristics to be Considered in Frequency Sharing Analyses Between GSO and NON-GSO Satellite Systems in the Fixed Satellite Service including Feeder Links for the Mobile-Satellite Service," ITU-R Recommendation S.1328-2, 2000.

[4] "Radio Regulations," International Telecommunication Union, 2001 .

[5] "Reference Earth-Station Radiation Pattern for Use in Coordination and Interference Assessment in the Frequency Range from 2 to About $30 \mathrm{GHz}$," ITU-R Recommendation S.465.

[6] "Radiation Diagrams for Use as Design Objectives for Antennas of Earth Stations Operating with Geostationary Satellites," ITU-R Recommendation S.580.

[7] "CCIR Report to the Second Session of the World Administrative Radio Conference on the Use of the GeostationarySatellite Orbit and the Planning of the Space Services Utilizing It (WARC-ORB(2))," Part I, pp. 44, Geneva 1988.

[8] "Proposal of Modification of the Recommends 6 of Recommendation ITU-R S.1323: GSO Earth Station Antenna Pattern to be Used for the Calculations of epfd," Document 4-9. II/156, ITU-R Joint Task Group 4-9-11 meeting, July 1998.
José Mauro P. Fortes received the E.E. (Telecommunications) degree in 1973 and the M.Sc.E.E. in 1976, both from Pontifícia Universidade Católica do Rio de Janeiro (PUC-Rio). He received later the M.Sc. and Ph.D. degrees from Stanford University, CA, USA in 1978 and 1980, respectively, returning to PUC-Rio in June 1980. There he is Associate Professor with an appointment at Centro de Estudos em Telecomunicações da Pontifícia Universidade Católica (CETUC). He took a sabbatical leave during 1992 at the General Electric Research and Development Center in Schenectady, NY, USA, where he was a researcher with the Ultrasound Group. Prof. Fortes has published various papers in national and international journals and conferences. He has also participated in several research projects and consulted for private companies and government agencies. He was Vice-President for Study Group 4 of the Radiocommunication Sector, Intenational Telecommunication Union (ITU) in Geneva. He was also president of the Brazilian Telecommunications Society from March 1996 through February 2000. His research interests include sattelite transmission, communication theory, estimation theory, and digital transmission.

Raimundo Sampaio-Neto received both the E.E. and the M.E.E. degrees from Universidade Católica do Rio de Janeiro (PUC-Rio) in 1975 and 1978, respectively, and the Ph.D. degree from University of Southern California (USC) in 1983. He was associated with the Communication Science Institute of the Department of Electrical Engineering at USC as a Post-Doctoral fellow from November 1983 to June 1984 with an appointment as a member of the technical staff of Axiomatic Corporation, Los Angeles. He is now a Researcher at CETUC and Associate Professor of the Department of Electrical at PUC-Rio, where he has been since July 1994. During 1991 he was Visiting Professor in the Department of Electrical Engineering at USC. Prof. Sampaio has participated in various projects and has consulted for several private companies and government agencies. He was co-organizer of the Session on Recent Results for the IEEE Workshop on Information Theory, 1992, Salvador. He has also served as Technical Program co-Chairman for IEEE Global Telecommunications Conference (Globecom'99) which took place at Rio de Janeiro in December 1999 and as a member of the technical committees of several national (SBT) and intenational (ITS) symposia of the Brazilian Telecommunications Society. He has been in office for two consecutive terms on the Board of Directors of the Brazilian Telecommunications Society as First Secretary (20002001) and as Vice-President of Development and Diffusion (20022003). He is a consultant for CAPES and CNPq. His fields of interest include communication theory, digital transmission, and satellite transmission. 